\title{
Associations of eating habits and the saliva microbiota in Finnish adolescents
}

\author{
Jannina Viljakainen $^{1,2}$, Sajan C. Raju ${ }^{1,2}$, Rejane Augusta de Oliveira Figueiredo ${ }^{1,2}$ \\ Heli Viljakainen $^{1,3}$, Eva Roos ${ }^{1,2}$, Elisabete Weiderpass ${ }^{1,4}$ and Trine B. Rounge ${ }^{1,5}$ \\ ${ }^{1}$ Folkhälsan Research Center, Helsinki, Finland, \\ ${ }^{2}$ Faculty of Medicine, University of Helsinki, Helsinki, Finland, \\ ${ }^{3}$ Department of Food and Environmental Sciences, University of Helsinki, Helsinki, Finland, \\ ${ }^{4}$ International Agency for Research on Cancer, World Health Organization, Lyon, France and \\ ${ }^{5}$ Department of Research, Cancer Registry of Norway, Oslo, Norway
}

\begin{abstract}
The composition of diet plays a vital role in maintaining health and may alter the human microbiota. The main focus to date has been on the association between diet and gut microbiota and only few studies have investigated the correlation between diet and the saliva microbiota. Our aim was to investigate whether eating habits and meal patterns are associated with the saliva microbiota. In this study, we included 842 adolescents aged 11-14 years from the Finnish Health in Teens (Fin-HIT) study cohort. Adolescents answered a web-based questionnaire, including information on eating habits, breakfast and dinner patterns at school. Questions related to diet were adapted to the age group. Previously three major eating habits were identified: healthy, unhealthy and fruit and vegetable avoiders. Saliva microbiota profiles were produced by $16 \mathrm{~S}$ (V3-V4) sequencing on the Illumina HiSeq platform. We found that the regular breakfast eaters had a higher diversity than the irregular breakfast eaters (Shannon index mean 2.27 vs. 2.21, ANCOVA; p-value = 0.026 and inverse Simpsońs index mean 6.21 vs. 5.70, ANCOVA; p-value $=0.004$ adjusted for gender, age, language, body mass index (BMI) categories, and sequencing depth). No associations were found between microbiota and eating habit groups, and between microbiota and dinner pattern groups. Microbial composition differed between the regular breakfast and irregular breakfast eaters (Bray-Curtis dissimilarity index, PERMANOVA; $\mathrm{p}=0.021$ ), but not between eating habit groups or dinner pattern groups. A high abundance of Prevotella was identified in the fruit and vegetable avoide? s, irregular breakfast and irregular dinner eaters. Our results indicate that having an irregular breakfast may shape the saliva microbiota diversity. Prevotella may be an indicator of avoiding veggies and skipping meals.
\end{abstract}

\section{Conflict of Interest}

There is no conflict of interest. 